\title{
Application of Wavelet Transform for Fault Diagnosis in Rotating Machinery
}

\author{
Hocine Bendjama, Salah Bouhouche, and Mohamed Seghir Boucherit
}

\begin{abstract}
Vibration analysis is essential in improving condition monitoring and fault diagnostics of rotating machinery. Many signal analysis methods are able to extract useful information from vibration data. Currently, the most of these methods use spectral analysis based on Fourier Transform (FT). However, these methods present some limitations; it is the case of non-stationary signals. In the present work, we are interested to the vibration signal analysis by the Wavelet Transform (WT). The WT is one of the most important methods for signal processing; it is especially suitable for non-stationary vibration measurements obtained from accelerometer sensors. The monitoring results indicate that the WT can diagnose the abnormal change in the measured data.
\end{abstract}

Index Terms_-Vibration analysis, fault diagnosis, rotating machinery, spectral analysis, wavelet transform

\section{INTRODUCTION}

Growing demand for higher performance, safety and reliability of industrial systems has increased the need for fault diagnosis. Fault diagnosis has been becoming more and more important for process monitoring. During the last two decades, various sensors have been developed and employed for condition monitoring and fault diagnosis, include; displacement, vibration, dynamic force, acoustic emission, temperature, etc.

The vibration signal analysis is one of the most important methods used for condition monitoring and fault diagnostics, because they always carry the dynamic information of the system. Effective utilization of the vibration signals, however, depends upon the effectiveness of the applied signal processing techniques for fault diagnostics. With the rapid development of the signal processing techniques, the analysis of stationary signals has largely been based on well-known spectral techniques such as: Fourier Transform (FT), Fast Fourier Transform (FFT) and Short Time Fourier Transform (STFT) [1], [2]. Unfortunately, the methods based on FT are not suitable for non-stationary signal analysis [3]. In addition, they are not able to reveal the inherent information of non-stationary signals. These methods provide only a limited performance for machinery diagnostics [4]. In order to solve these problems, Wavelet Transform (WT) has been

Manuscript received December 30, 2011; revised February 21, 2012.

H. Bendjama is with the Iron and Steel Applied Research Unit-CSC, BP 196, Annaba, Algeria, and the Process Control Laboratory, National Polytechnic Scool, BP 182, El-Harrach, Algiers, Algeria (e-mail: hocine bendjama@yahoo.fr).

S. Bouhouche is with the Iron and Steel Applied Research Unit-CSC, BP 196, Annaba, Algeria (e-mail: bouhouche11@yahoo.fr).

M. S. Boucherit is with the Process Control Laboratory, National Polytechnic Scool, BP 182, El-Harrach, Algiers, Algeria (e-mail: ms_boucherit@yahoo.fr). developed. WT is a kind of variable window technology, which uses a time interval to analyze the high frequency and the low frequency components of the signal [5], [6]. The data using WT can be decomposed into approximation and detail coefficients in a multiscale, presenting then a more effective tool for non-stationary signal analysis than the FT. Many studies present the applications of WT to decompose signals for improving the performance of fault detection and diagnosis in rotating machinery [7]-[10].

In this work, we propose to implement the WT for condition monitoring of rotating machinery. It is evaluated using the experimental measurements data in the cases of mass unbalance and gear fault. The main goal of this technique is to obtain more detailed information contained in the measured data.

The remainder of this paper is organized as follows. Section 2 presents WT method and its formulations. System and faults descriptions are presented in Section 3. The monitoring results are discussed in Section 4. Finally, Section 5 concludes our contributions.

\section{WAVELET TRANSFORM}

In the last 20 years, WT has grown at an explosive rate. Wavelets have appealed to scientists and engineers of many different backgrounds. WT has led to exciting applications in signal analysis and numerical analysis, and many other applications are being studied [11], [12].

WT is a time-frequency analysis technique. Due to its strong capability in time and frequency domain, it is applied recently by many researchers in rotating machinery. It decomposes a signal in both time and frequency in terms of a wavelet, called mother wavelet. The WT includes Continuous Wavelet Transform (CWT) and Discrete Wavelet Transform (DWT). Let $\mathrm{s}(\mathrm{t})$ is the signal; the CWT of $\mathrm{s}(\mathrm{t})$ is defined as

$$
\operatorname{CWT}(a, b)=1 /(\sqrt{|a|}) \int_{-\infty}^{\infty} s(t) \psi^{*}((t-b) / a) d t
$$

where $\psi^{*}(\mathrm{t})$ is the conjugate function of the mother wavelet $\psi(\mathrm{t})(2)$, a and $\mathrm{b}$ are the dilation (scaling) and translation (shift) parameters, respectively. The factor $1 / \sqrt{a}$ is used to ensure energy preservation.

$$
\psi(t)=1 /(\sqrt{a}) \psi((t-b) / a)
$$

The mother wavelet must be compactly supported and satisfied with the admissibility condition

$$
\int_{-\infty}^{+\infty}|\hat{\psi}(w)|^{2} /|w| d w<\infty
$$


where

$$
\hat{\psi}(w)=\int \psi(t) \exp (-j w t) d t
$$

The DWT is derived from the discretization of CWT. The most common discretization is dyadic. The DWT is given by

$$
\operatorname{DWT}(j, k)=1 /\left(\sqrt{2^{j}}\right) \int_{-\infty}^{\infty} s(t) \psi^{*}\left(\left(t-2^{j} k\right) / 2^{j}\right) d t
$$

where $\mathrm{a}$ and $\mathrm{b}$ are replaced by $2^{\mathrm{j}}$ and $2^{\mathrm{j}} \mathrm{k}, \mathrm{j}$ is an integer.

A very useful implementation of DWT, called multiresolution analysis [13], is demonstrated in fig. 1. DWT analyzes the signal at different scales. It employs two sets of functions, called scaling functions and wavelet functions [13], [14], which are associated with low pass and high pass filters, respectively. The discrete signal is passed through a high pass filter $(\mathrm{H})$ and a low pass filter $(\mathrm{L})$, resulting in two vectors at the first level; approximation coefficient (A1) and detail coefficient (D1) [15], [16]. Application of the same transform on the approximation (A1) causes it to be decomposed further into approximation (A2) and detail (D2) coefficients at the second level. Finally, the signal is decomposed at the expected level. The approximations are the high-scale, low-frequency components and the details are the low-scale, high-frequency components of the signal.

The wavelet decomposition for level 3 is illustrated in fig. 1. Each vector $A_{j}$ includes approximately, $N / 2^{j}$ coefficients, where $\mathrm{N}$ is the number of data points in the input signal $\mathrm{s}$, and provides information about a frequency band $\left[0, \mathrm{Fs} / 2^{\mathrm{j}+1}\right]$, where $\mathrm{Fs}$ is the sampling frequency. In fig. $1 \mathrm{H}$ and $\mathrm{L}$ represent the decomposition filters, and $\downarrow 2$ denotes a down sampling by a factor of 2. An important property of the DWT is:

$$
\mathrm{s}=\mathrm{A} 3+\mathrm{D} 1+\mathrm{D} 2+\mathrm{D} 3
$$

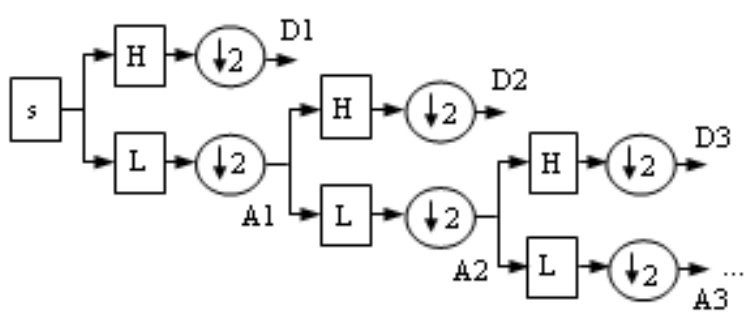

Fig. 1. Principle of DWT decomposition.

\section{EXPERIMENTAL SETUP}

\section{A. System Description}

The measurement of vibration applied to condition monitoring and fault diagnosis requires different types of equipment and techniques. These depend on the investment and available expertise. It is essential to have a mean of analysis of vibration which may consist of the following: in addition to the studied structure itself, sensors, data collectors and the analyzer.

The experiment equipment used throughout this paper performs the condition monitoring of rotating machinery. It consists of two gears 1 and 2 ( 1 with 60 teeth and the other with 48 teeth), four bearing housings ( $\mathrm{H} 1, \mathrm{H} 2, \mathrm{H} 3$ and $\mathrm{H} 4)$, coupling and disk, as shown in fig. 2 . The system is driven by a $0.18 \mathrm{~kW}$ induction motor, giving an output of 0-1500 rpm, controlled by a variable speed drive. In order to predict any anomalies that may occur under different measurement conditions, we collected real vibration signals from this experimental system. The vibration signals were taken on bearing housing $\mathrm{H} 1$ through a piezoelectric accelerometer measured the radial vibration. These measurements were repeated for different states of the system at different rotation speeds of the motor. The data acquisition was performed using the OROS25 software. Each measured vibration signal is available over a window of 400 milliseconds. Two kinds of faults were simulated in this work; mass unbalance and gear fault.

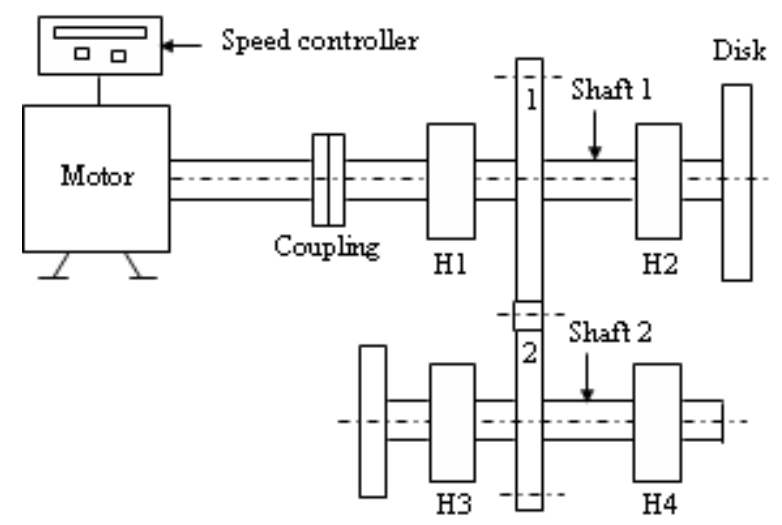

Fig. 2. Illustration of experimental system.

\section{B. Faults Description}

\section{1) Mass unbalance}

Mass unbalance is one of the most common causes of vibration; it is simulated in our application by an additional weight on the disk. Unbalance is a condition where the centre of mass does not coincide with the centre of rotation, due to the unequal distribution of the mass about the centre of rotation. The unbalance creates a vibration frequency exactly equal to the rotational speed, with amplitude proportional to the amount of unbalance (see fig. 3).

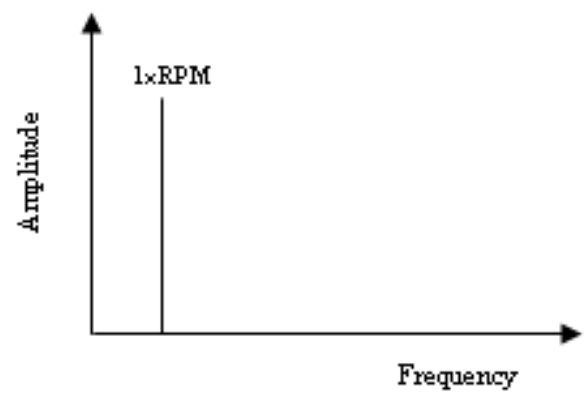

Fig. 3. Frequency spectrum of mass unbalance.

\section{2) Gear fault}

The vibrations of a gear are mainly produced by the shock between the teeth of the two wheels. Gear fault is simulated with filled between teeth. The vibration monitored on a faulty gear generally exhibits a significant level of vibration at the tooth meshing frequency GMF (i.e. the number of teeth on a 
gear multiplied by its rotational speed) and its harmonics of which the distance is equal to the rotational speed of each wheel (see fig. 4).

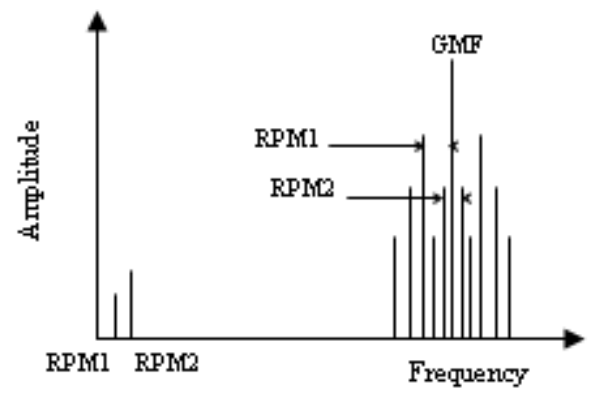

Fig. 4. Frequency spectrum of gear fault.

\section{Results AND Discussions}

The vibration signals used in this work were gained through the practical measurement. They were taken in radial direction at different rotating speeds, including a mass unbalance and a gear fault. The number of data points for each signal is 2048 .

Fig. 5 represents the vibration signal in time domain and its spectrum. The signal is collected from the mass unbalance at a speed of $1200 \mathrm{rpm}(20 \mathrm{~Hz})$ on bearing housing $\mathrm{H} 1$ of the shaft 1 only i.e. without meshing. It is not easy to detect fault from this figure.

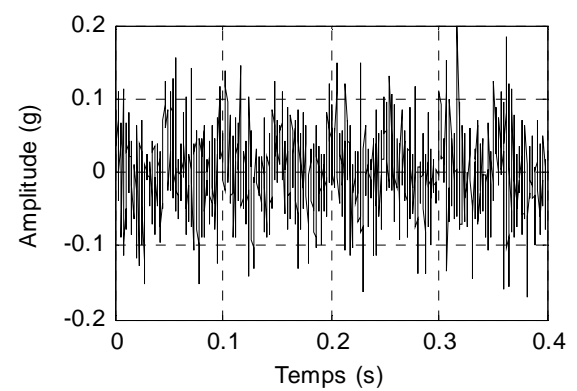

(a)

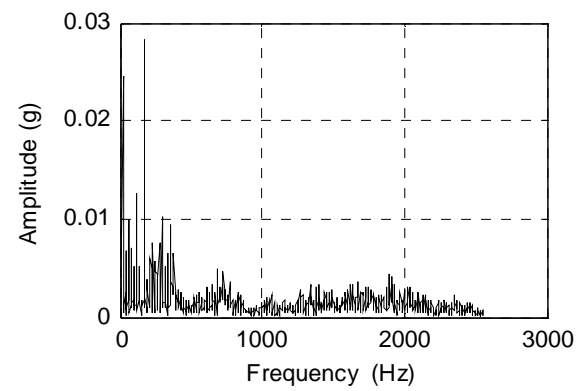

(b)

Fig. 5. Vibration signal of mass unbalance and its spectrum.

The vibration signal of the gear fault measured at a speed of $600 \mathrm{rpm}(10 \mathrm{~Hz})$ in time and frequency domain is shown in fig. 6. Obviously, there is no particularly clear demonstration i.e. the characteristic gear fault frequency is not clear from the frequency spectrum.

The identification and the monitoring of the mass unbalance and the gear fault using the spectral analysis are difficult, due to the non-stationary. To extract the fault information, the WT will be applied to the vibration signals.
The selection of the appropriate wavelet is very important in signals analysis. There are many functions available can be used such as Haar, Daubechies, Meyer, and Morlet functions [17]. Different wavelets serve different purposes. In this application, we used the Daubechies wavelet [18] and the Morlet wavelet [19], [20] for the fault diagnosis of mass unbalance and gear, respectively.

The multiresolution analysis is applied by using the Daubechies wavelet of order $4(\mathrm{db} 4)$. In which level 4 decomposition is employed to extract the approximation coefficient from vibration signals. The result of $\mathrm{db} 4$ decomposition of the vibration signal of the mass unbalance collected at a rotation speed of $1200 \mathrm{rpm}$ is given in fig. 7 .

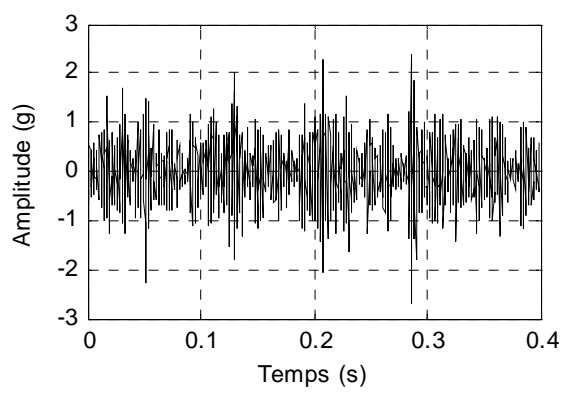

(a)

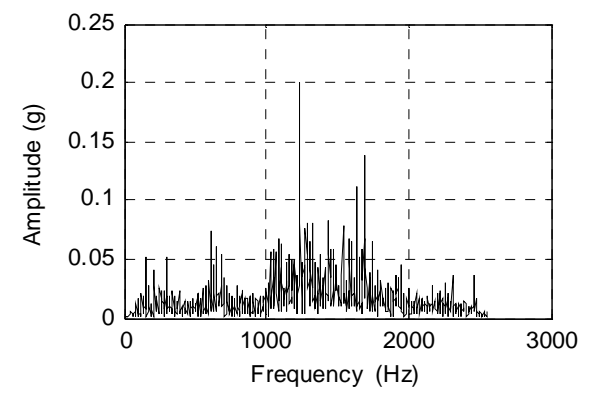

(b)

Fig. 6. Vibration signal of gear fault and its spectrum.
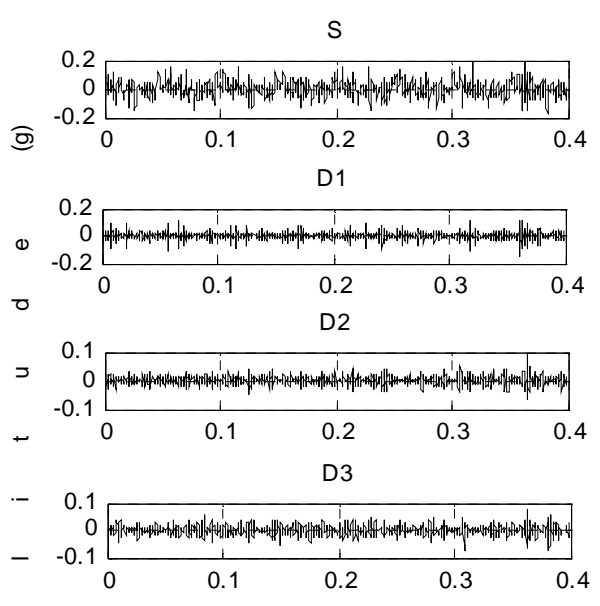

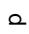

$\therefore$ D4

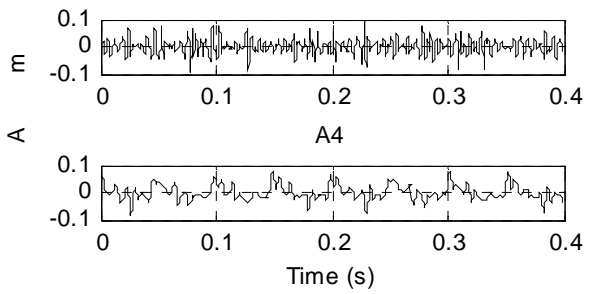

Fig. 7. Decomposition of vibration signal of mass unbalance at a speed of $1200 \mathrm{rpm}$ with db4 wavelet. 
Fig. 8 shows the approximation 4 (A4) and its spectrum. A frequency peak at $20 \mathrm{~Hz}$ is present, which could be related to a mass unbalance fault, and its harmonics $(40,60,80 \mathrm{~Hz} .$. could be identified, these frequency components are well differentiated from the adjacent frequency in the spectrum, confirming the presence of a mass unbalance fault.

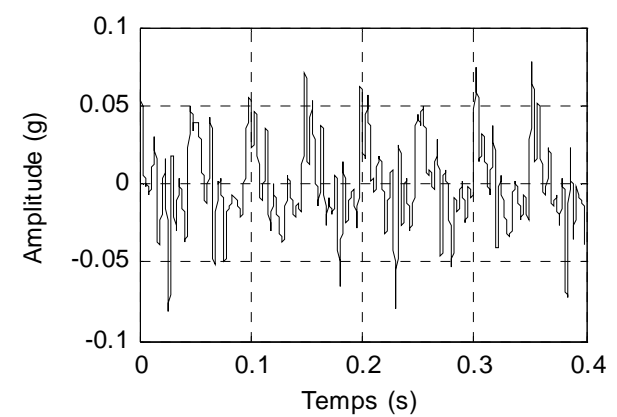

(a)

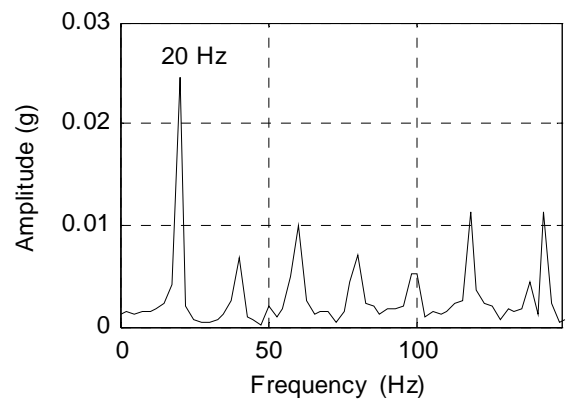

(b)

Fig. 8. A4 of mass unbalance and its spectrum.
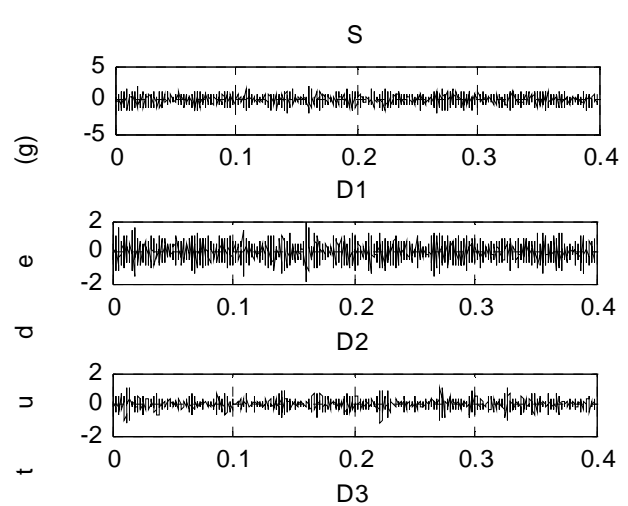

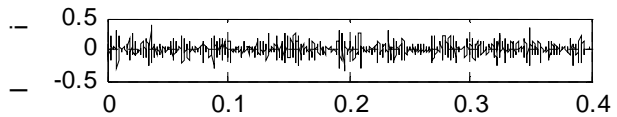

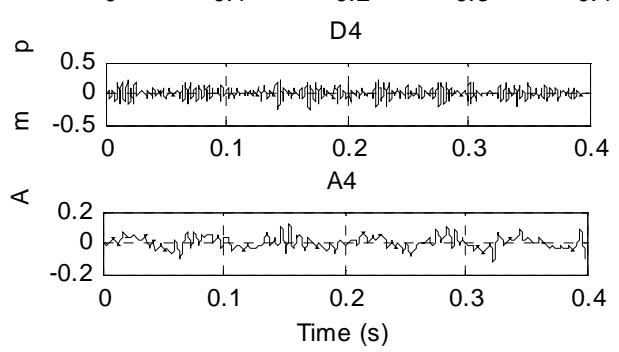

Fig. 9. Decomposition of vibration signal of mass unbalance at a speed of $900 \mathrm{rpm}$ with db4 wavelet.

Fig. 9, and 10 show the vibration signals of the mass unbalance collected at a speed of 900 and $1125 \mathrm{rpm}$ (15 and
18.75 Hz) and the corresponding approximation and detail coefficients (obtained by db4) up to four levels. In this case the vibration signals are measured on bearing housing $\mathrm{H} 1$ of the shaft 1 meshed with the shaft 2. Fig. 11 and 12 represent the FT of A4. It is clear that the peaks at 15 and $18.75 \mathrm{~Hz}$ are present; this is due to a mass unbalance fault, which is consistent with the theory.

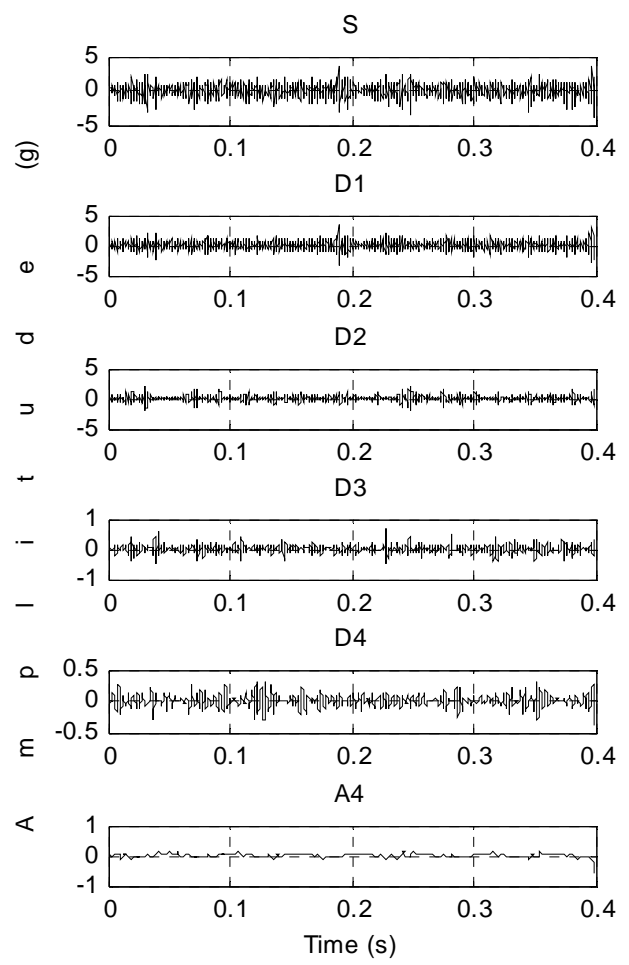

Fig. 10. Decomposition of vibration signal of mass unbalance at a speed of $1125 \mathrm{rpm}$ with db4 wavelet.

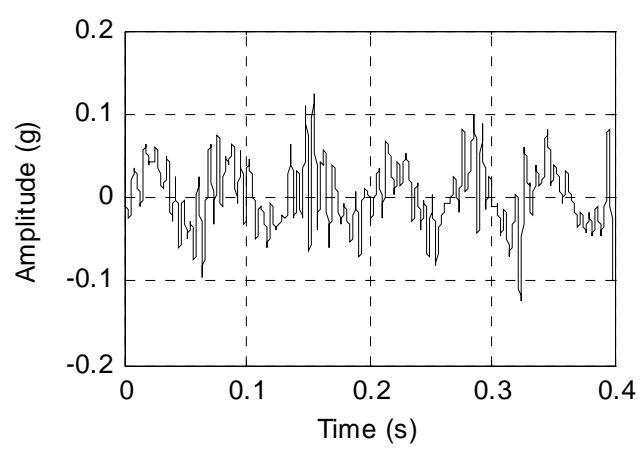

(a)

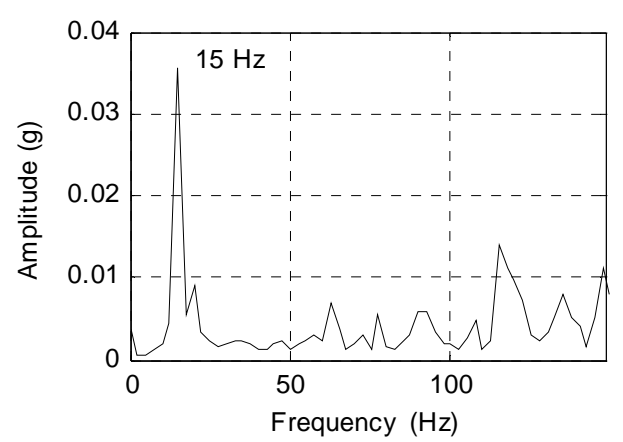

(b)

Fig. 11. A4 of mass unbalance and its spectrum. 


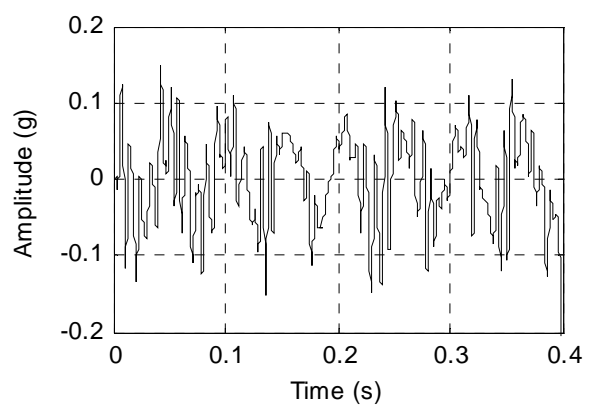

(a)

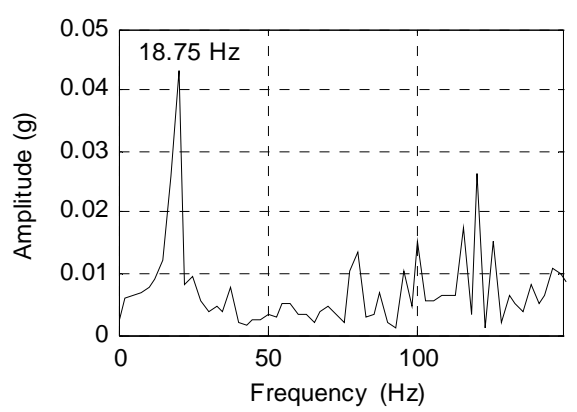

(b)

Fig. 12. A4 of mass unbalance and its spectrum.

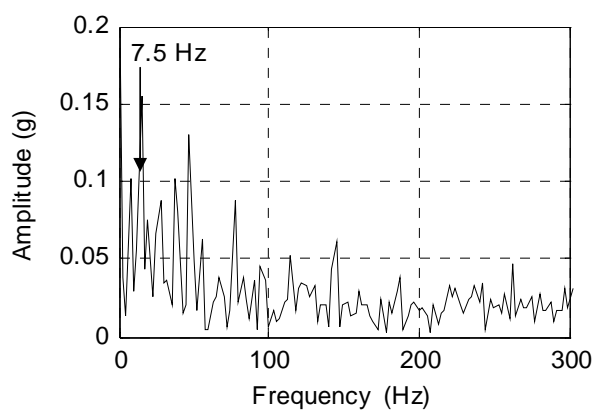

(a)

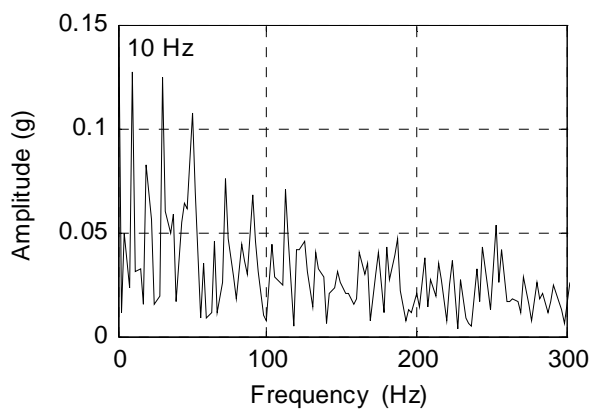

(b)

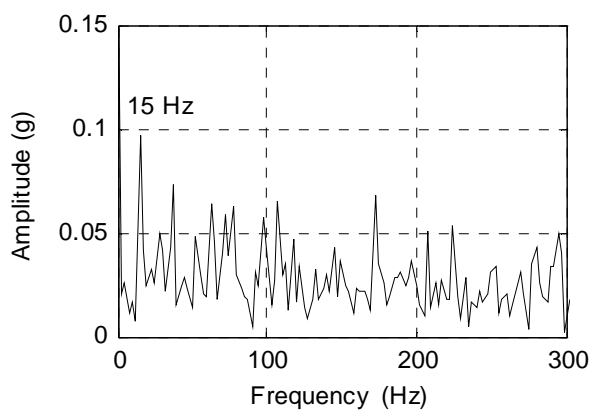

(c)

Fig. 13. Spectrum of gear fault obtained with Morlet wavelet.
The identification of the gear fault is possible by using the Morlet wavelet, it is also called CWT. It can be seen from fig. 13 that the peaks at the rotation frequencies of the shaft (7.5, 10 and $15 \mathrm{~Hz}$ ) and its multiples are present in the frequency spectrum. This clearly indicates a gear fault.

\section{CONCLUSION}

This paper presents a fault diagnosis method based on the WT. DWT and CWT have been tested on real measurement signals collected from a vibration system containing mass unbalance and gear fault. Better results are obtained by identifying the type of fault. Such method is useful for improving the conditions monitoring and faults diagnosis of rotating machines. It remains to test its application on a signal containing two or more types of faults.

\section{REFERENCES}

[1] K. Shibata, A. Takahashi, T. Shirai, "Fault diagnosis of rotating machinery through visualisation of sound signal," Journal of Mechanical Systems and Signal Processing, vol. 14, pp. 229-241, 2000.

[2] S. Seker, E. Ayaz, "A study on condition monitoring for induction motors under the accelerated aging processes," IEEE Power Engineering, vol. 22, no. 7, pp. 35-37, 2002.

[3] J. C. Cexus, "Analyse des signaux non-stationnaires par Transformation de Huang, Opérateur de Teager-Kaiser, et Transformation de Huang-Teager (THT)," Thèse de Doctorat, Université de Rennes-France, 2005.

[4] J. D. Wu, C.-H. Liu, "Investigation of engine fault diagnosis using discrete wavelet transform and neural network," Expert Systems with Applications, vol. 35, pp. 1200-1213, 2008.

[5] S. H. Cao, J. C. Cao, "Forecast of solar irradiance using recurrent neural networks combined with wavelet analysis," Applied Thermal Engineering, vol. 25, no. 2-3, pp. 161-172, 2005.

[6] G. Strang, "Wavelet transforms versus Fourier transforms," Bulletin of the American Mathematical Society, vol. 28, pp. 288-305, 1993.

[7] Z. K. Peng, F. L. Chu, "Application of the wavelet transform in machine condition monitoring and fault diagnostics: a review with bibliography," Mechanical Systems and Signal Processing, vol. 18, pp. 199-221, 2004.

[8] S. Prabhakar, A. R. Mohanty, A. S. Sekhar, "Application of discrete wavelet transform for detection of ball bearing race faults," Tribology International, vol. 35, pp. 793-800, 2002.

[9] C. K. Sung, H. M. Tai, C. W. Chen, "Locating defects of a gear system by the technique of wavelet transform," Mechanism and Machine Theory, vol. 35, pp. 1169-1182, 2000.

[10] A. Djebala, N. Ouelaa, N. Hamzaoui, "Detection of rolling bearing defects using discrete wavelet analysis," Meccanica, vol. 43, no. 2, pp. 339-348, 2008.

[11] I. Daubechies, Ten Lectures on Wavelets, Philadelphia, PA: SIAM, 1992.

[12] Jianguo Yang. "An anti-aliasing algorithm for discrete wavelet transform," Mechanical Systems and Signal Processing, vol. 17, no. 5, pp. 945-954, 2003.

[13] S. G. Mallat, "A theory for multiresolution signal decomposition: the wavelet representation," IEEE Trans Pattern Anal Machine Intelligence, vol. 11, no. 7, pp. 674-693, 1989.

[14] N. Lu, F. Wang, F. Gao, "Combination method of principal component and wavelet analysis for multivariate process monitoring and fault diagnosis," Ind. Eng. Chem. Res, vol. 42, pp. 4198-4207, 2003.

[15] D. E. Newland, "Wavelet analysis of vibration, part I: theory, part II: wavelet maps," Journal of Vibration and Acoustics, vol. 116, pp. 409-416, 1994.

[16] D. E. Newland, "Wavelet analysis of vibration, part I: theory, part II: wavelet maps," Journal of Vibration and Acoustics, vol. 116, pp. 417-425, 1994.

[17] Ch. K Chui, An Introduction to Wavelets, Academic Press: New York, 1992. 
[18] I. Daubechies, "Orthonormal bases of compactly supported wavelets," Communication on Pure and Applied Mathematics, vol. 41, pp. 909-996, 1988

[19] P. Goupillaud, A. Grossmann, J. Morlet, "Cycle-octave and related transforms in seismic signal analysis," Geoexploration, vol. 23, pp. 85-102, 1984.

[20] P. S. Addison, J. N. Watson, T. Feng, "Low-oscillation complex wavelets," Journal of Sound and Vibration, vol. 254, no. 4, pp. 733-762, 2002

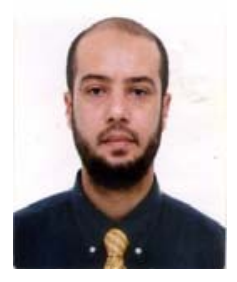

Hocine Bendjama was born in Constantine, Algeria, in 1974. He received the Engineer degree and the Magister degree in electronics from the University of Science and Technology, Mentouri, Constantine, Algeria in 2000 and 2003 respectively. In 2007, he joined the Industrial Monitoring Laboratory of Iron and Steel Applied Research Unit (URASM/CSC) Annaba, Algeria. He is currently a Ph.D student in Process Control Laboratory of Polytechnic National School Algiers, Algeria. His research interests include system modelling and optimization, process fault diagnostics, and neural networks.

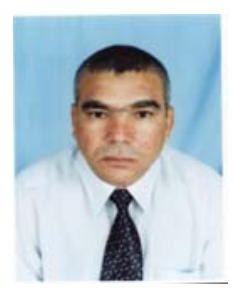

Salah Bouhouche was born in Collo/Skikda, Algeria, in 1960. Graduated from Algerian Institute of Hydrocarbon and Chemistry-Boumerdes Algeria in 1985. In 1995 he obtained his Magister degree in electrical engineering from the Polytechnic National School. In 2002, he received his Ph.D degree in maschinenbau from TU Bergakademie Freiberg, Germany. In 2005, he obtained a DSC (habilitation) in electrical engineering from the Polytechnic National School Algiers, Algeria. He is a senior researcher in the field of quality and process monitoring, control and optimization of the industrial processes in the Iron and Steel Applied Research Unit (URASM/CSC).

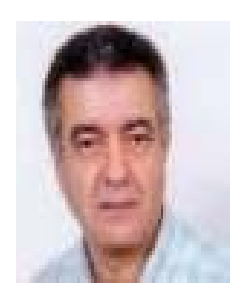

Mohamed Seghir Boucherit was born in 1954 in Algiers, Algeria. He received the Engineer degree in Electrotechnics, the Magister degree and the Ph.D degree in electrical engineering, from the Polytechnic National School, Algiers, Algeria, in 1980, 1988 and 1995 respectively. Upon graduation, he joined the Electrical Engineering Department of Polytechnic National School. He is Professor, member of Process Control Laboratory and his research interests are in the area of electrical drives and process control. 